\title{
Editorial
}

\section{Non-linearity in Natural and Social Sciences}

\author{
E. VELIKHOV and A. KINGSEP* \\ Russian Research Center, Kurchatov Institute, 123182, Moscow,Russia
}

(Revised 3 January 2001)

During the last few decades non-linear sciences, the first of which being non-linear physics, have come to be the subject of the most advanced treatment among the natural sciences. One of the great scientific achievements of the 19th century was the development of the universal approach which considered a variety of phenomena, such as oscillations and waves, transport phenomena or diffusive processes. Many effects and processes in physics, mechanics, and chemistry began to be considered on a common basis and with some common understanding, regardless of their particular nature.

Based on this common understanding, mutual enrichment took place. One example was Volterra's equation in mathematical physics, which was initially constructed to describe the interaction of a community of predators and their victims. Such a unification of understanding dealt mainly with natural sciences, while socio-political processes remained at the level of the humanities or the mathematically naïve. We now observe some progress in this direction. First, economics and life sciences are involved in mutual enrichment. Secondly, one of the more important recent scientific developments is the formation of nonlinear science. Initially it may seem to be only a mathematical term, that is, non-linear science is any science to which a non-linear approach applies. In fact, there exists a vast group of non-linear effects which are no less universal than the linear phenomena described in the 19th century. The enrichment noted above is a non-symmetric one; some basic results and methods are transferred from physics to chemistry and then to biology, life sciences and social and economic sciences. Going in the opposite direction, the problem depends on the methods of verification, since the more fundamental the natural science, the more defined are the models.

Unlike in linear science, the variety of non-linear phenomena can hardly be studied and properly described analytically (although some analytical results play an important role, serving as a basis for universal scenarios). Moreover, in economics any reasonable approach must be based on numerical methods. In order to understand today's socio-political realities, one has to deal with large amounts of information, which must be analyzed by computer.

*Corresponding author. 
That is why numerical methods, together with nonlinear physics, provide the basis for non-linear science.

Many phenomena in life and social sciences were incompatible with the typical characteristics of linear phenomena, i.e. oscillations, diffusive processes, and entropy growth, which reveal their limitations. Now we recognize that most phenomena in these areas are essentially non-linear. In particular, one of the main features of any self-consistent, non-linear dynamic is the formation of non-linear structures. We can observe these structures in mechanics (solitons and vortices, strange attractors), chemistry (Belousov-Zhabotinsky reactions), dynamics of the Earth's crust, biology, and the complicated dynamics of human history and world economy. Many of their features when properly described appear to be universal.
Our goal is to examine the laws that apply to nonlinear phenomena, starting from physics and transferring them to build reasonable scenarios in both life and social sciences, using an extrinsic method. Such a treatment is complementary to traditional methods developed specifically for each of these sciences. As we know from the physics and chemistry developed in the 20th century, the complementary approach turns out to be fruitful in natural sciences (part of which is human society).

Hereafter, some papers devoted to these problems are presented. Despite their being very different from each other, they all follow the general idea declared in the title. 


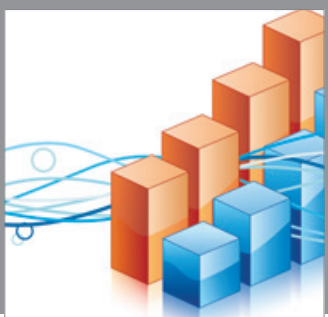

Advances in

Operations Research

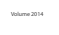

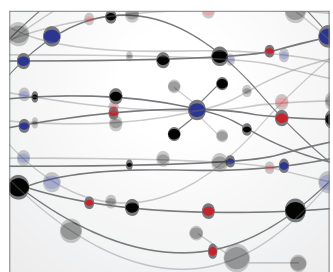

\section{The Scientific} World Journal
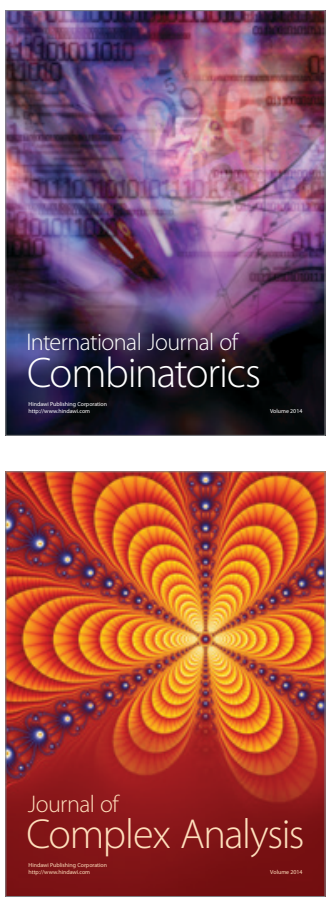

International Journal of

Mathematics and

Mathematical

Sciences
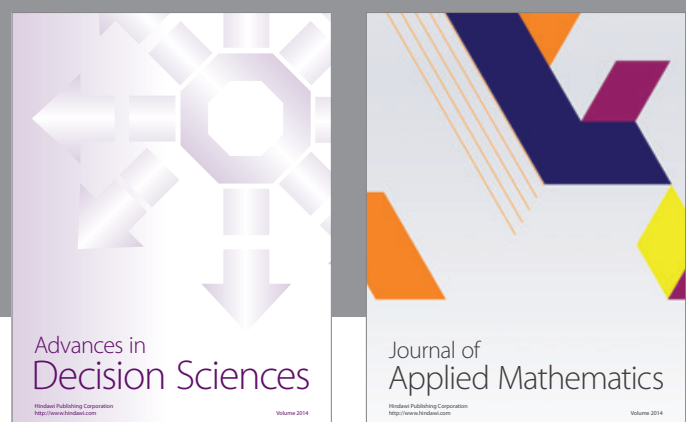

Journal of

Applied Mathematics
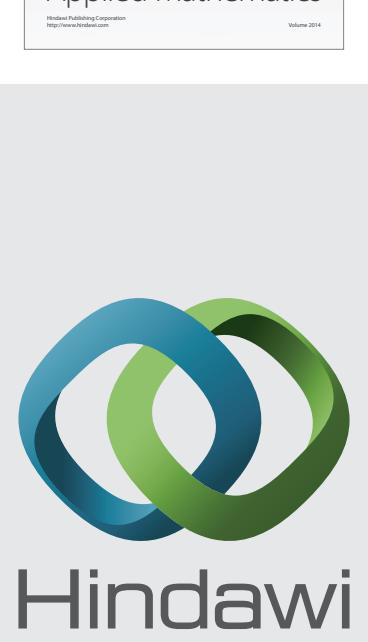

Submit your manuscripts at http://www.hindawi.com
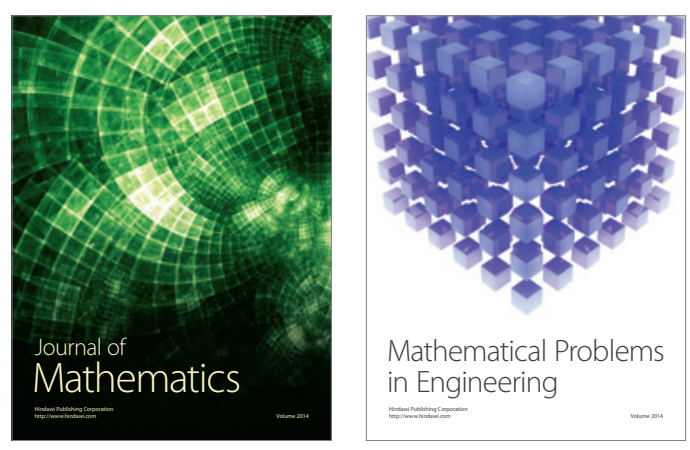

Mathematical Problems in Engineering
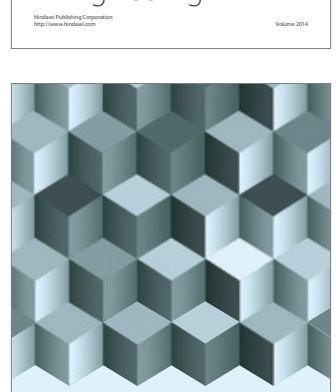

Journal of

Function Spaces
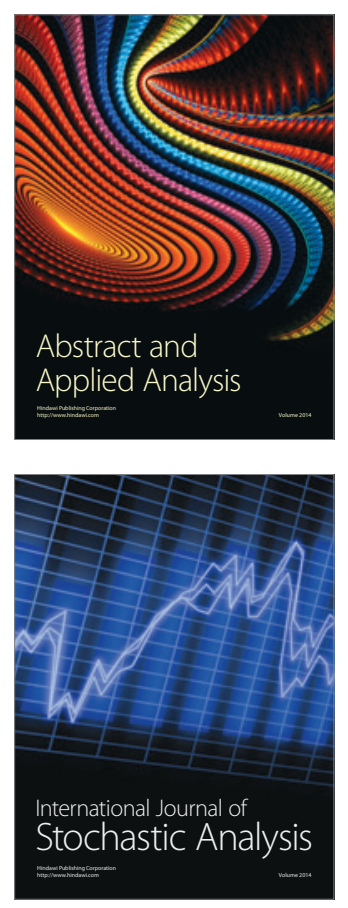

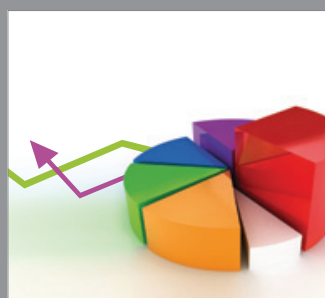

ournal of

Probability and Statistics

Promensencen
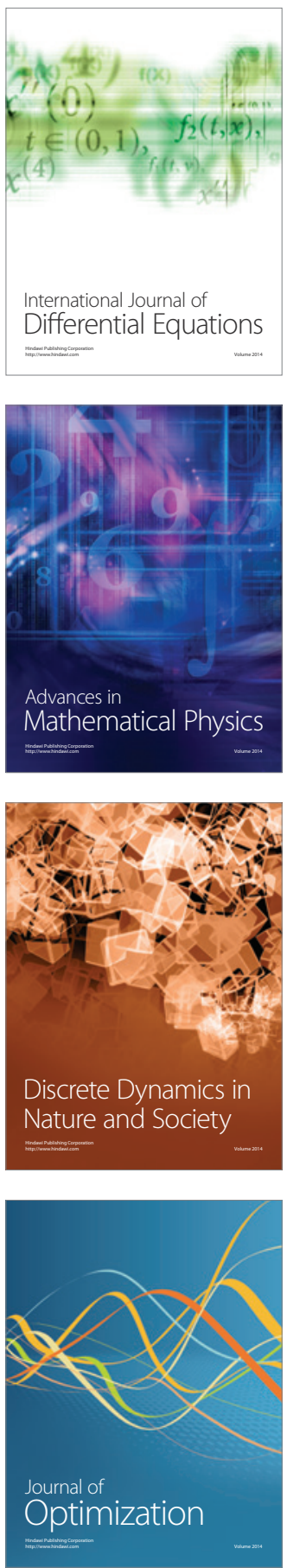